\title{
CRECIMIENTO RADIAL Y CONICIDAD DEL FUSTE EN PLANTACIONES CON ACLAREO DE Pinus patula SCHIEDE ex SCHLECHTENDAL \& CHAMISSO
}

\author{
RADIAL GROWTH AND STEM TAPERING IN THINNED PLANTATIONS OF \\ Pinus patula SCHIEDE ex SCHLECHTENDAL \& CHAMISSO
}

\author{
Luz Ma. Reyes-Cortés ${ }^{1}$, J. Jesús Vargas-Hernández ${ }^{1}$, \\ Arnulfo Aldrete ${ }^{1}$ y J. Amador Honorato-Salazar ${ }^{2}$
}

\begin{abstract}
'Colegio de Postgraduados, Campus Montecillo, Postgrado en Ciencias Forestales, Montecillo, Texcoco, Estado de México, México. ${ }^{2} \mid$ nstituto Nacional de Investigaciones Forestales, Agrícolas y Pecuarias, Campo Experimental San Martinito, Tlahuapan, Puebla, México.
\end{abstract}

*Autor de correspondencia (vargashj@colpos.mx)

\section{RESUMEN}

Pinus patula Schiede ex Schlechtendal \& Chamisso es una especie endémica de México, con gran relevancia económica por su alto potencial productivo en plantaciones forestales y bosques naturales. La intensidad y frecuencia de las cortas de aclareo generalmente influyen en el grueso de los anillos anuales de crecimiento, la proporción de madera tardía y la conicidad del fuste. El objetivo del presente estudio fue evaluar el crecimiento radial y el grueso de los anillos de crecimiento y de sus componentes de madera temprana y tardía a diferentes alturas del fuste, así como el índice de conicidad del fuste en plantaciones jóvenes de Pinus patula con cortas de aclareo aplicadas a los nueve y 13 años de edad. Se seleccionaron y derribaron 10 árboles por parcela con diámetro normal similar al valor promedio de éstas, para obtener tres secciones transversales (rodajas) de $3 \mathrm{~cm}$ de grueso a diferentes alturas de cada árbol muestreado. Las cortas de aclareo causaron un impacto reducido en el grueso de los anillos anuales y sus componentes de madera temprana y tardía en la sección basal. El efecto de la corta se presentó sólo al año siguiente de su aplicación, con incrementos en el área basal y en el grueso de madera temprana; después, el ritmo de crecimiento radial regresó a su patrón original. El grueso de los anillos y de la madera temprana y tardía fue mayor en la parte intermedia y superior del fuste, lo que ocasionó una reducción gradual en la conicidad, lo cual corroboró que la aplicación de las cortas de aclareo fueron tardías, cuando la copa ya se había concentrado en la parte superior del árbol, por la elevada competencia por espacio de crecimiento.

Palabras clave: Pinus patula, anillos de crecimiento, competencia, madera tardía, madera temprana, perfil del fuste.

\section{SUMMARY}

Pinus patula Schiede ex Schlechtendal \& Chamisso is an endemic species of Mexico, with significant economic relevance due to its high productive potential in forest plantations and natural forests. The intensity and frequency of thinning cuts generally influence the thickness of the annual growth rings, the proportion of latewood, and the tapering of the stem. This study evaluated the radial growth and thickness of the growth rings and their earlywood and latewood components at different stem heights, as well as the stem taper index of young Pinus patula plantations with thinning cuts applied at nine and 13 years of age. Ten trees were selected and cut in each of two thinned plots with a diameter at breast height similar to that of the plot mean to obtain three $3-\mathrm{cm}$ thick cross sections (slices) at different heights of each sampled tree. Thinning cuts reduced the impact on the thickness of the annual rings and their earlywood and latewood components at the basal section. The effect of the cut was only observable the year after the thinnings, with an increase in basal area and earlywood thickness; afterwards, the radial growth rate returned to its original pattern. The thickness of the rings and their earlywood and latewood components was higher at the middle and upper stem sections, causing a gradual reduction in stem tapering. This results confirmed that thinning cuts were performed late, when the crown had already concentrated at the top of the tree, due to high competition for growth space.

Index words: Pinus patula, competition, earlywood, growth rings, latewood, stem taper.

\section{INTRODUCCIÓN}

Pinus patula es una especie endémica de México con gran relevancia económica por su alto potencial productivo para el establecimiento de plantaciones de crecimiento rápido. El árbol presenta copa abierta y redondeada, fuste recto y madera de calidad; es ampliamente utilizado en plantaciones en Sudáfrica, India, Australia y Sudamérica, donde se ha introducido (Dvorak et al., 2000). En México se distribuye en forma natural en las regiones de la Sierra Madre Oriental, Eje Neovolcánico y Sierra Madre de Oaxaca (Sánchez-González, 2008).

La madera, principal finalidad del cultivo de esta especie, se origina del proceso biológico de la actividad mitótica del cambium secundario, que incluye la división y expansión celular, diferenciación, maduración, muerte celular programada y formación del duramen dentro del mismo árbol para constituir el tejido del xilema (Plomion et al., 2001). Se ha demostrado que la actividad cambial presenta sensibilidad alta a diferentes factores ambientales; por lo tanto, si se presentan cambios en alguno de los factores, como la competencia o la disponibilidad de agua, se modifica la dinámica estacional en los procesos de diferenciación celular y formación de la madera, con efectos positivos o negativos sobre la actividad del cambium (Gruber et al., 2010). 
La formación de la madera depende de factores ambientales, genéticos y fisiológicos; entre los ambientales, las condiciones del sitio y las prácticas silvícolas ocasionan efectos en el crecimiento de los árboles y formación de madera, al modificar la temperatura, la radiación y la disponibilidad de agua y nutrientes, así como el nivel de competencia entre los árboles (Balducci et al., 2013; Swidrak et al., 2011). La modificación en la fenología y tasa de crecimiento influye en las características anatómicas del xilema, lo que conduce a diferencias en la amplitud de los anillos de crecimiento, la cantidad de madera temprana y tardía, y en la densidad de la madera a lo largo del eje longitudinal del árbol (Larson, 1969).

A través de las prácticas silvícolas, como los aclareos (cortas intermedias), es posible modificar las condiciones de desarrollo y afectar las características de los anillos de crecimiento (Rodríguez-Ortiz et al., 2011b). Después de aplicar un aclareo, los árboles residuales tienden a reaccionar al espaciamiento entre ellos y se presenta un aumento en la velocidad de crecimiento; debido a esto, el aclareo es la principal herramienta para el control de la densidad del arbolado, lo cual modifica los patrones de partición de biomasa (Rodríguez-Ortíz et al., 2011a); sin embargo, la aplicación tardía o ligera de una corta de aclareo puede reducir el grueso de los anillos, aumentar la proporción de madera tardía y ocasionar una forma más cilíndrica del fuste (Larson et al., 2001). A largo plazo es importante evaluar la respuesta del crecimiento de los árboles residuales a las cortas de aclareo, en particular el efecto en el crecimiento radial y la proporción de madera temprana y tardía en los anillos anuales, que afectan la calidad de la madera (Hernández et al., 1996; Vásquez et al., 2016). Los objetivos de este trabajo fueron evaluar el crecimiento radial y el grosor de los anillos (madera temprana y tardía) a diferentes alturas del fuste, así como la conicidad del fuste en plantaciones de Pinus patula Schiede ex Schlechtendal \& Chamisso con cortas de aclareo aplicadas a los nueve y 13 años de edad.

\section{MATERIALES Y MÉTODOS}

\section{Área de estudio}

El estudio se realizó en una plantación de Pinus patula establecida en 1995 en la comunidad de Ixtlán de Juárez, Oaxaca, México, en las coordenadas geográficas de $17^{\circ} 22^{\prime}$ $38.3^{\prime \prime} \mathrm{N}$ y $96^{\circ} 28^{\prime} 43.2^{\prime \prime} \mathrm{O}$, con altitud promedio de $2538 \mathrm{~m}$. En la evaluación se incluyeron dos parcelas permanentes de monitoreo del crecimiento de $400 \mathrm{~m}^{2}(20 \times 20 \mathrm{~m})$. A cada parcela se le aplicaron cortas de aclareo con diferente intensidad para propósitos de investigación en los años 2004 y 2008. El historial de manejo y las características dasométricas de las parcelas se presentan en el Cuadro 1.

\section{Muestreo en campo}

El derribo de los árboles se realizó en marzo de 2017, se tomaron muestras (rodajas) del fuste de 10 árboles por parcela para análisis del crecimiento radial a diferentes alturas del fuste. Los árboles seleccionados tuvieron dimensiones cercanas al diámetro promedio por parcela (24 cm en la Parcela 1 y $26 \mathrm{~cm}$ en la Parcela 2). Del fuste se extrajeron tres secciones transversales (rodajas) de $3 \mathrm{~cm}$ de grueso. La primera rodaja $\left(\mathrm{S}_{1}\right)$ se obtuvo en la base, a 30 $\mathrm{cm}$ del suelo, la segunda $\left(\mathrm{S}_{2}\right)$ de la base de la tercera troza comercial y la tercera rodaja $\left(S_{3}\right)$ del extremo distal de la última troza comercial (tercera o cuarta troza, en función de la altura total del árbol).

Cuadro 1. Historial de manejo y características dasométricas de las parcelas de Pinus patula.

\begin{tabular}{|c|c|c|c|c|c|c|c|}
\hline \multirow{2}{*}{$\begin{array}{l}\text { Año de aclareo/ } \\
\text { Edad }^{+}\end{array}$} & \multirow{2}{*}{$\begin{array}{l}\text { Características } \\
\text { dasométricas }\end{array}$} & \multicolumn{3}{|c|}{ Parcela 1} & \multicolumn{3}{|c|}{ Parcela 2} \\
\hline & & Inicial & Residual & IA (\%) & Inicial & Residual & $\mathrm{IA}(\%)$ \\
\hline \multirow{4}{*}{ 2004/9 años } & NA & 3600 & 1400 & & 3650 & 2650 & \\
\hline & $\mathrm{DN}(\mathrm{cm})$ & 10.40 & 16.30 & & 9.70 & 10.80 & \\
\hline & AB $\left(m^{2} h a^{-1}\right)$ & 38.27 & 30.06 & 21.5 & 30.25 & 26.32 & 13.0 \\
\hline & $\operatorname{VOL}\left(\mathrm{m}^{3} \mathrm{ha}^{-1}\right)$ & 145.98 & 123.93 & & 112.10 & 99.88 & \\
\hline \multirow{4}{*}{ 2008/13 años } & NA & 1400 & 1250 & & 2650 & 2200 & \\
\hline & $\mathrm{DN}(\mathrm{cm})$ & 20.10 & 20.70 & & 15.30 & 16.20 & \\
\hline & $\mathrm{AB}\left(\mathrm{m}^{2} \mathrm{ha}^{-1}\right)$ & 46.02 & 43.25 & 6.0 & 52.06 & 47.87 & 8.1 \\
\hline & $\operatorname{VOL}\left(\mathrm{m}^{3} \mathrm{ha}^{-1}\right)$ & 298.37 & 280.45 & & 281.86 & 261.89 & \\
\hline
\end{tabular}

${ }^{\dagger}$ Año y edad de los árboles al momento del aclareo. IA: intensidad del aclareo en AB, NA: número de árboles, DN: diámetro normal, AB: área basal, VOL: volumen del fuste (Fuente: Rodríguez-Ortiz et al., 2012). 
Antes de derribar el árbol se marcó la dirección norte del fuste para medir las rodajas en los puntos cardinales; éstas se identificaron y transportaron al laboratorio de Ciencias Forestales del Colegio de Postgraduados para ser almacenadas a la sombra, en ambiente fresco (temperatura menor de $20^{\circ} \mathrm{C}$ ) durante $15 \mathrm{~d}$, para permitir la pérdida de humedad; después, las rodajas se seccionaron en forma de cruz con $4 \mathrm{~cm}$ de ancho. La sección principal se mantuvo en dirección norte-sur; por último, se pulieron a mano con lijas de agua (grano de 240 y 1000) hasta ver con claridad los anillos de crecimiento.

\section{Medición de árboles vecinos}

Durante el muestreo de campo se identificaron y midieron los cuatro árboles vecinos más cercanos al árbol objetivo (árbol medio) para estimar la densidad, se establecieron los cuadrantes de acuerdo con la orientación cardinal (N, E, S y O) y se midió el diámetro normal (DN), la altura total y la distancia con respecto al árbol objetivo. Con estos datos se calculó un índice de competencia para cada árbol derribado $\left(\mathrm{IC}_{\mathrm{i}}\right)$ mediante la expresión siguiente (Daniels et al., 1986):

$$
I C_{i}=\Sigma\left(D N_{j} / d_{i j}\right)
$$

donde: $\mathrm{I} \mathrm{C}_{\mathrm{i}}=$ índice de competencia del i-ésimo árbol objetivo, $\mathrm{DN}_{\mathrm{j}}=$ diámetro normal del j-ésimo árbol vecino, $\mathrm{d}_{\mathrm{ij}}=$ distancia del j-ésimo árbol vecino al i-ésimo árbol objetivo.

\section{Procesamiento de muestras en laboratorio}

Las rodajas lijadas fueron escaneadas (Escaner Epson® modelo Perfection 4990, Suwa, Japón) y cada imagen digitalizada se procesó con el software WinDENDROß (versión 2009). Se contaron los anillos de crecimiento por radio; se tomó como referencia el último anillo formado en el año 2016; se identificó el año de formación de cada anillo, se midió su grueso total (GA), grueso de madera temprana (GTE) y de madera tardía (GTA). Con estos registros se obtuvo la proporción de madera tardía (PT) de cada anillo de crecimiento anual.

El incremento anual en diámetro y área basal se estimó con el grueso de los anillos de crecimiento para cada radio y sección del árbol. Con estos datos se obtuvo el índice de conicidad de cada árbol para evaluar el efecto de los aclareos sobre el incremento en diámetro a lo largo del perfil del fuste. El índice de conicidad relaciona el diámetro en diferentes secciones del fuste. Se obtuvo la relación de las secciones $S_{2}$ y $S_{3}$ con respecto a $S_{1}$ (es decir, $S_{2} / S_{1}$ y $S_{3} /$ $S_{1}$ ), así como la relación $S_{3} / S_{2}$. Dado que las rodajas de la sección superior $\left(S_{3}\right)$ se extrajeron de diferente altura del fuste, se realizó un ajuste del diámetro a una misma altura con los valores del diámetro acumulado, tras suponer una reducción gradual por cada metro de longitud con base en la reducción del diámetro en la tercera troza del árbol correspondiente.

\section{Análisis estadístico}

Se realizó análisis de varianza con el procedimiento GLM del paquete estadístico SAS, versión 9.4 (SAS Institute, 2016) para evaluar el efecto de las cortas de aclareo aplicadas a los nueve y 13 años de edad en las variables medidas en los anillos de crecimiento. El análisis se hizo por separado en cada sección del fuste. Se realizó un análisis nuevo utilizando valores relativos de las características de los anillos.

El valor relativo de cada variable a evaluar (grueso de los anillos de crecimiento) se obtuvo mediante la razón entre los valores absolutos y su valor promedio en cada árbol y parcela, con la finalidad de unificar las unidades de medida del grueso de los anillos o incremento radial entre árboles dentro de una misma parcela. El uso de los valores relativos permitió identificar en cada árbol los anillos con valores menores y mayores al promedio y ajustar los datos por las diferencias en tamaño y crecimiento entre ellos. La ecuación utilizada para obtener los valores relativos fue:

$$
X_{i j r}=\left(X_{i j} / \bar{X}_{j}\right) \times \bar{X}
$$

Donde: $X_{\mathrm{ij} r}=$ valor relativo de la variable respuesta en el i-ésimo anillo del $\mathrm{j}$-ésimo árbol, $\mathrm{X}_{\mathrm{ij}}=$ valor absoluto de la variable respuesta en el i-ésimo anillo del j-ésimo árbol, $\bar{X}$ $=$ valor promedio del j-ésimo árbol y $\bar{X}=$ valor promedio de la variable respuesta de la parcela.

Con los valores del índice de conicidad por árbol se aplicó análisis de varianza con el procedimiento GLM de SAS para evaluar diferencias entre parcelas en estas variables por efecto de los aclareos realizados.

\section{RESULTADOS Y DISCUSIÓN}

\section{Anillos de crecimiento en la base del árbol}

El análisis de varianza con valores absolutos de los anillos de crecimiento mostró diferencias no significativas $(P>0.05)$ entre las parcelas, pero al usar valores relativos de las mismas variables las diferencias fueron significativas $(P \leq 0.05)$ en el incremento en área basal y en el grueso de madera temprana (Cuadro 2). Aunque no hubo un efecto significativo de los aclareos en GA (con excepción del año 2010), en IAB y GTE sí se detectó el efecto de los aclareos de 2004 y 2008, especialmente en el periodo de 
crecimiento de 2005 a 2010.

Los árboles residuales de las dos parcelas con diferente intensidad de corta tuvieron el mismo patrón de respuesta en las variables de los anillos (Figura 1a). En ambos casos, los anillos fueron gruesos en los primeros años, y después el grueso de la madera temprana y tardía disminuyó de manera gradual y se estabilizó en los últimos años. El incremento en área basal (IAB) fue influenciado por la edad y la competencia por el espacio de crecimiento; tuvo un aumento rápido en los primeros ocho años y posteriormente declinó (Figura 1b). A diferencia de las variables anteriores, la proporción de madera tardía aumentó gradualmente con la edad, aunque con ligeras reducciones en algunos años (Figura 1e). Los árboles residuales de la Parcela 1 presentaron valores mayores en las características de los anillos de crecimiento después de aplicar un aclareo más intenso (21.5 \% en AB) a los nueve años de edad, lo que dejó menor número de árboles en pie, pero con diámetro promedio mayor.

En general, las cortas de aclareo ocasionan un incremento en el grueso de los anillos de los árboles residuales con la apertura del dosel y menor competencia por espacio de crecimiento (Koga et al., 1997). En diferentes estudios se ha encontrado que el efecto en el grueso de los anillos es mayor al aumentar la intensidad del aclareo (Zhang et al., 2006); por ejemplo, Pamerleau-Couture et al. (2015) encontraron en Picea mariana que el incremento radial de los árboles residuales aumentó de 0.07 a $0.14 \mathrm{~cm}$ después de la aplicación del aclareo con una intensidad de corta de 30 a $35 \%$ de área basal.

Se esperaba encontrar un aumento en el grosor de los anillos de crecimiento por efecto de los aclareos realizados a los nueve y 13 años de edad, especialmente en los árboles de la Parcela 1, donde el primer aclareo removió $21.5 \%$ del AB. El aclareo aplicado en 2008 sólo tuvo impacto en el comportamiento del grosor de los anillos en 2010, aunque en el caso del área basal el efecto fue más duradero, con diferencias entre las dos parcelas incluso cuatro o cinco años después del segundo aclareo.

El impacto reducido de los aclareos sobre las características de los anillos de crecimiento en Pinus patula se puede deber a que éstos se aplicaron demasiado tarde (Niemistö et al., 2018), si se considera la densidad inicial de la plantación y el elevado nivel de competencia desde su establecimiento. Las parcelas presentaron 3600 o más árboles ha-1 antes del aclareo de 2004, lo que se

Cuadro 2. Valor de probabilidad (P) en características de anillos de crecimiento en la base del árbol de Pinus patula con cortas de aclareo.

\begin{tabular}{|c|c|c|c|c|c|c|c|c|c|c|}
\hline \multirow{2}{*}{$\begin{array}{l}\text { Año de } \\
\text { formación }\end{array}$} & \multicolumn{5}{|c|}{ Valores absolutos } & \multicolumn{5}{|c|}{ Valores relativos } \\
\hline & GA & IAB & GTE & GTA & PT & GA & IAB & GTE & GTA & PT \\
\hline 2004 & 0.839 & 0.531 & 0.924 & 0.756 & 0.966 & 0.832 & 0.564 & 0.737 & 0.900 & 0.801 \\
\hline 2005 & 0.240 & 0.031 & 0.227 & 0.366 & 0.786 & 0.103 & 0.011 & 0.047 & 0.072 & 0.615 \\
\hline 2006 & 0.477 & 0.110 & 0.274 & 0.813 & 0.319 & 0.329 & 0.043 & 0.848 & 0.148 & 0.323 \\
\hline 2007 & 0.399 & 0.111 & 0.970 & 0.281 & 0.305 & 0.207 & 0.014 & 0.050 & 0.872 & 0.273 \\
\hline 2008 & 0.508 & 0.205 & 0.522 & 0.572 & 0.819 & 0.424 & 0.114 & 0.325 & 0.448 & 0.613 \\
\hline 2009 & 0.243 & 0.096 & 0.349 & 0.420 & 0.608 & 0.100 & 0.004 & 0.120 & 0.336 & 0.513 \\
\hline 2010 & 0.081 & 0.042 & 0.067 & 0.146 & 0.284 & 0.046 & 0.008 & 0.041 & 0.050 & 0.358 \\
\hline 2011 & 0.825 & 0.479 & 0.722 & 0.786 & 0.442 & 0.734 & 0.198 & 0.864 & 0.712 & 0.289 \\
\hline 2012 & 0.288 & 0.149 & 0.194 & 0.986 & 0.116 & 0.236 & 0.051 & 0.678 & 0.687 & 0.039 \\
\hline 2013 & 0.118 & 0.080 & 0.106 & 0.193 & 0.774 & 0.075 & 0.020 & 0.073 & 0.203 & 0.713 \\
\hline 2014 & 0.304 & 0.176 & 0.194 & 0.488 & 0.768 & 0.258 & 0.105 & 0.214 & 0.512 & 0.795 \\
\hline 2015 & 0.124 & 0.074 & 0.094 & 0.120 & 0.769 & 0.110 & 0.042 & 0.150 & 0.105 & 0.736 \\
\hline 2016 & 0.200 & 0.095 & 0.194 & 0.030 & 0.010 & 0.247 & 0.122 & 0.327 & 0.028 & 0.016 \\
\hline
\end{tabular}

GA: grueso de anillo, IAB: incremento de área basal, GTE: grueso de madera temprana, GTA: grueso de madera tardía, PT: proporción de madera tardía. Los números en negritas indican probabilidades significativas. 

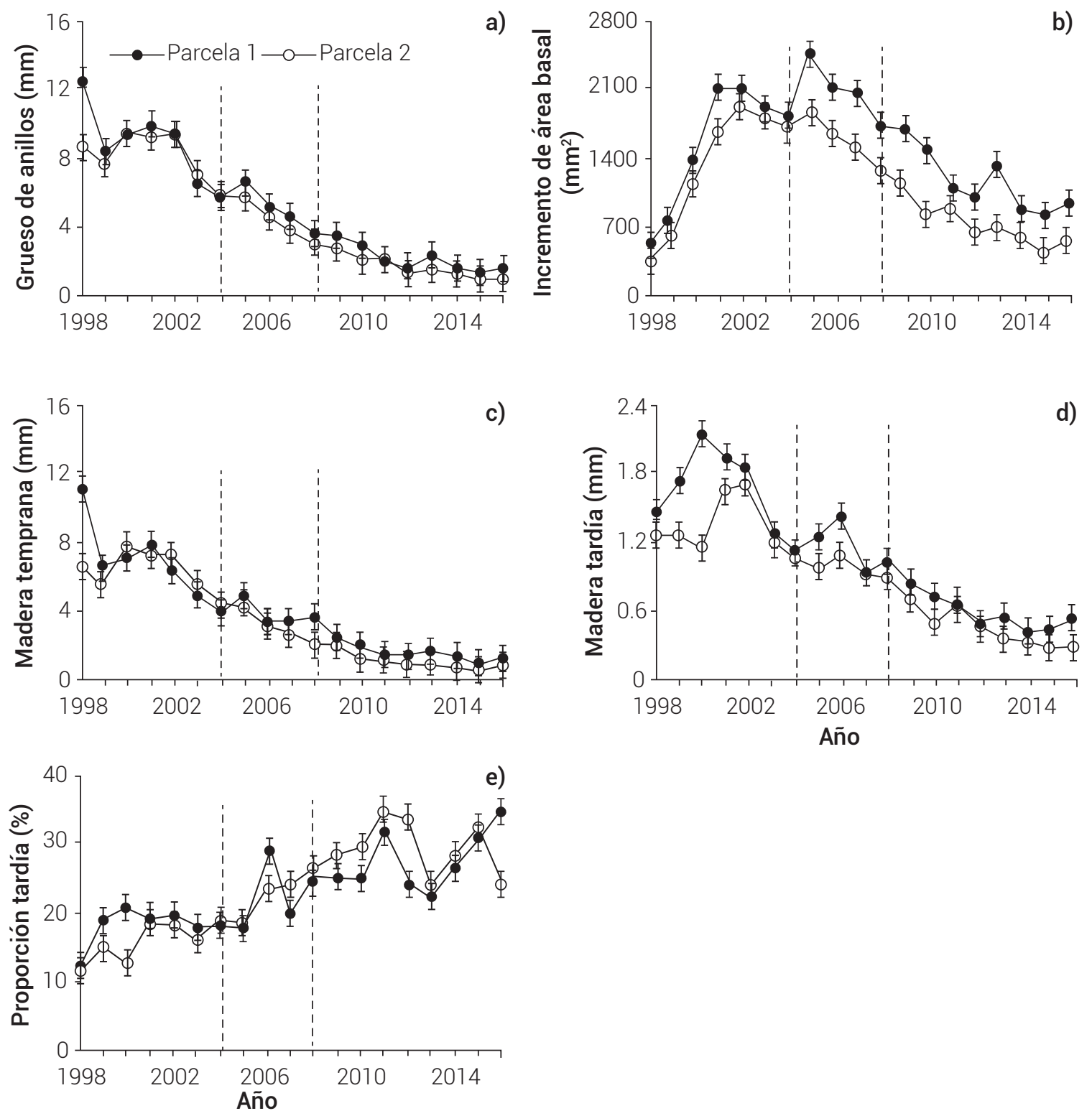

Figura 1. Respuesta en características de los anillos de crecimiento en la sección basal de Pinus patula en parcelas con diferente intensidad de aclareo (21.5\% y $13.0 \%$ de AB a los nueve años; $6.0 \%$ y $8.1 \%$ a los 13 años de edad). Las líneas verticales indican el momento de los aclareos. a) grueso de anillo, b) incremento en área basal, c) grueso de madera temprana, d) grueso de madera tardía, e) proporción de madera tardía.

reflejó en un diámetro normal promedio relativamente bajo, sobre todo si se considera que la tasa de crecimiento promedio de la especie y las condiciones ambientales del área de estudio son favorables (Rodríguez-Ortiz et al., 2012); además, el patrón de las características de los anillos de crecimiento mostró que dos a tres años antes de aplicar el primer aclareo, se inició una reducción acelerada en el grosor del anillo y de sus características asociadas de madera temprana y tardía.
A pesar que en la Parcela 1 se eliminó el $60 \%$ de los árboles, el efecto sobre las características de los anillos fue casi nulo a largo plazo, pero fue significativo en el área basal con respecto a la Parcela 2, donde el número de árboles eliminados fue menor de $30 \%$. La elevada competencia inicial previa al aclareo redujo el tamaño de la copa viva y el crecimiento radial en la parte baja del fuste, como se ha encontrado en otras especies de coníferas 
(Fabris, 2000; Com. Pers)' ${ }^{1}$ Estos factores explican el efecto reducido del aclareo realizado a los 13 años de edad, cuando la intensidad de corta fue menor de $10 \%$ en AB en ambas parcelas (aclareo ligero) y la copa se había retraído todavía más por la competencia de espacio en la

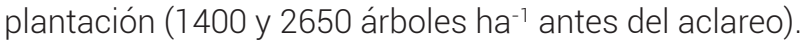

\section{Anillos de crecimiento a diferentes alturas del fuste}

Las características de los anillos de crecimiento en las secciones intermedia y superior del fuste mostraron diferencias significativas $(P \leq 0.05)$ entre parcelas con cortas de aclareo sólo en los primeros años de formación. En el periodo 2008-2010 se encontraron diferencias en incremento del área basal, en madera temprana y proporción de madera tardía en la sección intermedia. En la sección superior las diferencias fueron en el grueso del anillo y de la madera temprana y madera tardía en 2012 y 2014 (Cuadro 3).

El patrón de respuesta de las variables grosor de los anillos de crecimiento en las secciones intermedia y superior del fuste fue similar a la sección basal (Figura 2). Cabe señalar que se encontró mayor grosor de anillos y de madera temprana en la parcela con mayor número de árboles residuales; lo anterior indica que posiblemente las diferencias son por el efecto de la posición de la copa en los árboles y no por el efecto de la corta de aclareo. La respuesta común de los árboles a una corta de aclareo es el aumento en el crecimiento radial a lo largo de todo el

\footnotetext{
'Fabris S. (2000) Influence of cambial ageing, initial spacing, stem taper and growth rate on the wood quality of three coastal conifers. Doctoral Thesis. University of British Columbia. Vancouver, Canada. 266 p.
}

fuste, pero en particular en la parte baja, debido al mayor tamaño de copa que mantienen los árboles al reducirse la competencia lateral (Tong et al., 2011; Winck et al., 2015).

Estudios realizados con diferentes intensidades de poda de la copa muestran que al reducir el tamaño de la copa viva ocurre un desplazamiento hacia arriba del fuste en la actividad del cambium, lo que modifica el patrón de crecimiento radial del árbol a lo largo de su eje debido a la menor disponibilidad de carbohidratos en la parte baja del árbol (Balducci et al., 2013; Ferrere et al., 2015).

En este estudio el patrón manifestó que el grueso del anillo en un mismo año de crecimiento es menor en la parte baja del fuste que en la parte intermedia o superior. El crecimiento radial de los árboles de Pinus patula en el periodo de 2008 a 2016 es dos veces mayor en las secciones intermedia y superior que en la sección basal, y el efecto fue mayor en la Parcela 2, donde hay mayor competencia por espacio, lo que corrobora este desplazamiento en el perfil longitudinal de la actividad del cambium asociado con la reducción en el tamaño de la copa de los árboles, como lo han señalado Gartner et al. (2002) y Gruber et al. (2010).

A pesar que el grueso de los anillos en las secciones intermedia y superior fue mayor en árboles de la Parcela 2, el incremento en área basal fue similar, o incluso superior, en la Parcela 1; ésto se debe a que los árboles de la Parcela 1 tuvieron mayor altura promedio al momento de realizar las cortas de aclareo, y por lo tanto, tenían en la sección intermedia y superior más anillos de crecimiento acumulados previos al primer año de medición, lo que

Cuadro 3. Valor de probabilidad $(P)$ en características de anillos de crecimiento en la sección intermedia y superior del árbol de Pinus patula con cortas de aclareo.

\begin{tabular}{|c|c|c|c|c|c|c|c|c|c|c|}
\hline \multirow{2}{*}{$\begin{array}{l}\text { Año de } \\
\text { formación }\end{array}$} & \multicolumn{5}{|c|}{ Sección intermedia } & \multicolumn{5}{|c|}{ Sección superior } \\
\hline & GA & IAB & GTE & GTA & PT & GA & $\mathrm{IAB}$ & GTE & GTA & PT \\
\hline 2008 & 0.289 & 0.002 & 0.230 & 0.592 & 0.341 & & & & & \\
\hline 2009 & 0.201 & 0.014 & 0.205 & 0.561 & 0.608 & & & & & \\
\hline 2010 & 0.170 & 0.022 & 0.239 & 0.258 & 0.873 & & & & & \\
\hline 2011 & 0.047 & 0.902 & 0.028 & 0.637 & 0.013 & & & & & \\
\hline 2012 & 0.606 & 0.049 & 0.252 & 0.085 & 0.073 & 0.004 & 0.406 & 0.048 & 0.037 & 0.960 \\
\hline 2013 & 0.316 & 0.392 & 0.244 & 0.843 & 0.761 & 0.009 & 0.270 & 0.015 & 0.327 & 0.352 \\
\hline 2014 & 0.989 & 0.081 & 0.759 & 0.503 & 0.340 & 0.022 & 0.034 & 0.042 & 0.006 & 0.994 \\
\hline 2015 & 0.375 & 0.063 & 0.464 & 0.473 & 0.741 & 0.899 & 0.069 & 0.076 & 0.245 & 0.411 \\
\hline 2016 & 0.912 & 0.561 & 0.714 & 0.749 & 0.401 & 0.473 & 0.440 & 0.487 & 0.489 & 0.668 \\
\hline
\end{tabular}

GA: grueso de anillo, IAB: incremento de área basal, GTE: grueso de madera temprana, GTA: grueso de madera tardía, PT: proporción de madera tardía. Los números en negritas indican probabilidades significativas. 

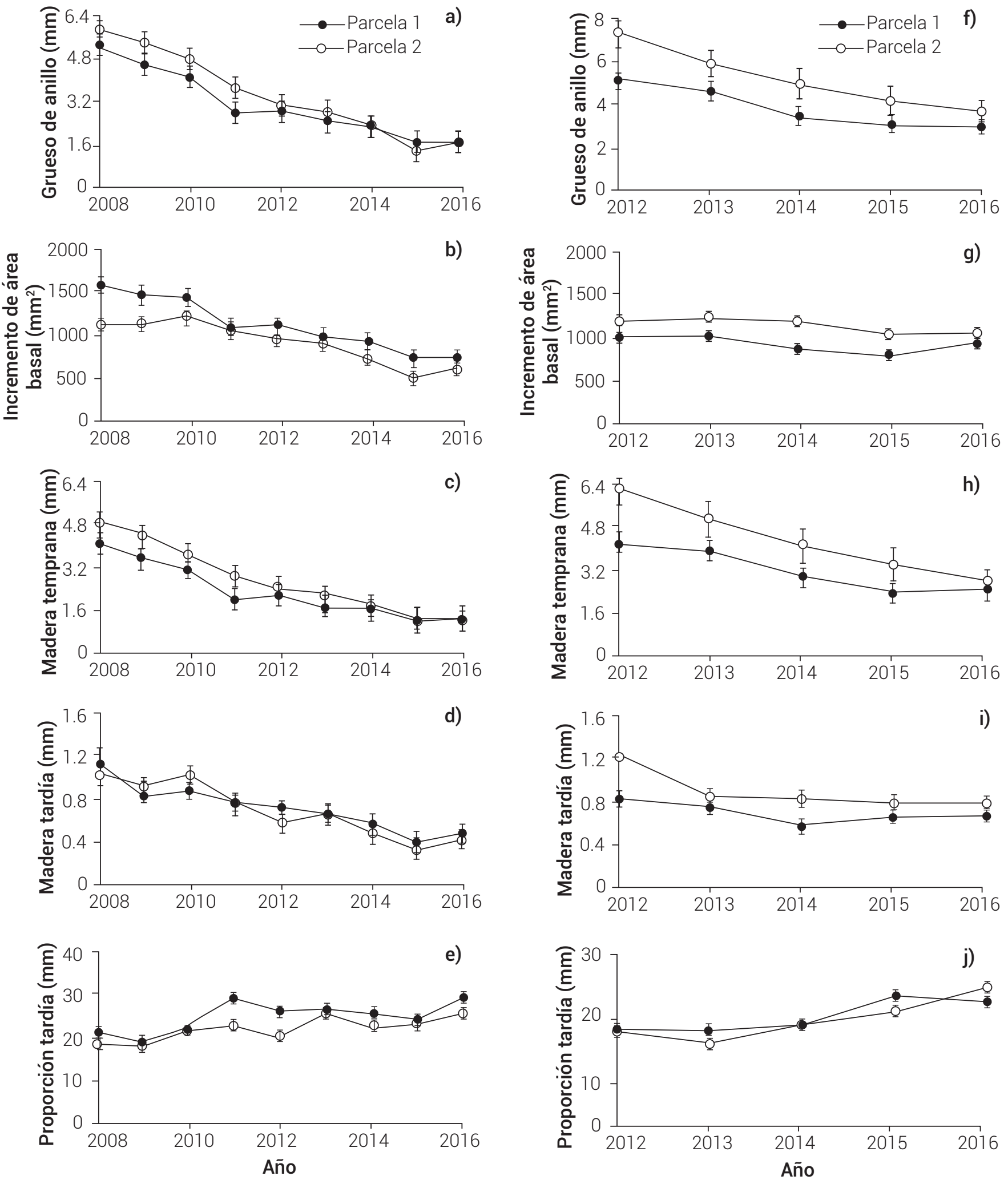

Figura 2. Respuesta en características de los anillos de crecimiento en la sección intermedia (a-e) y superior (f-j) de fuste de Pinus patula en parcelas con diferente intensidad de aclareo: $a, f$ ) grueso de anillo; b, g) incremento en área basal; c, h) grueso de madera temprana; d, i) grueso de madera tardía; e, j) proporción de madera tardía. 
ocasionó mayor área en la corona circular representada por el anillo correspondiente.

\section{Efecto de la corta de aclareo en la conicidad del fuste}

El análisis de varianza mostró diferencias no significativas $(P>0.05)$ entre las parcelas con cortas de aclareo en el índice de conicidad del fuste, estimado a partir del diámetro acumulado en las secciones transversales a diferentes alturas del mismo (Cuadro 4).

La conicidad del fuste se redujo gradualmente con la edad de los árboles en las dos parcelas (Figura 3) por el mayor grueso y acumulación relativa de los anillos de crecimiento en las secciones intermedia y superior del fuste. El índice de conicidad permite evaluar el grado de conicidad (o su complemento, cilindricidad) del fuste de los árboles. La mayoría de los estudios con cortas de aclareo enfatizan sus efectos en el crecimiento individual del arbolado o del rodal; al aumentar el espacio de crecimiento y reducir la competencia se espera un crecimiento mayor de los árboles y redistribución de la capacidad productiva del sitio entre el arbolado residual (Pape, 1999); sin embargo, también se espera que la corta ocasione aumento en la conicidad del árbol; es decir, se reduzca el valor del índice de conicidad, al aumentar la longitud de copa y el crecimiento radial en la base del árbol; en cambio, las podas ocasionan reducción de la conicidad al generar desplazamiento de la actividad cambial y mayor crecimiento radial en la parte superior del árbol (Fernández et al., 2017; Pinkard y Beadle, 2000).

Durante el periodo de estudio, los árboles de ambas parcelas aumentaron gradualmente en $I C_{21}, I_{31}$ e IC $C_{32}$ hasta alcanzar valores de $0.56,0.22$ y 0.40 , respectivamente, lo que implica una reducción gradual de la conicidad del fuste. Este patrón es característico del efecto ocasionado por tratamientos de poda de la copa (Fernández et al., 2017) y refleja, sin duda, la respuesta en el patrón longitudinal del crecimiento radial de los árboles debido a la reducción drástica del tamaño de la copa por la excesiva competencia de los árboles, antes y después de las cortas de aclareo; es decir, los aclareos se aplicaron tarde y no se removió el número suficiente de árboles para revertir el proceso de recesión de la copa ocasionada por la elevada competencia de espacio en la plantación.

En un estudio realizado en bosques manejados de Pinus patula se demostró que el índice de conicidad del árbol varía con la intensidad del manejo de la densidad, con valores de 0.44 en bosque natural, 0.46 en bosque con manejo intensivo y de 0.50 en plantaciones con mayor control de la competencia (Uranga-Valencia et al., 2015).

\section{CONCLUSIONES}

Las cortas de aclareo aplicadas a los nueve y 13 años de edad en árboles de Pinus patula tuvieron un impacto reducido en estimular el crecimiento radial del fuste

\begin{tabular}{|c|c|c|c|}
\hline Años de formación & $I_{21}$ & $\mathrm{IC}_{31}$ & $\mathrm{IC}_{32}$ \\
\hline 2004 & 0.491 & - & - \\
\hline 2005 & 0.704 & - & - \\
\hline 2006 & 0.368 & - & - \\
\hline 2007 & 0.335 & - & - \\
\hline 2008 & 0.311 & - & - \\
\hline 2009 & 0.294 & 0.161 & 0.486 \\
\hline 2010 & 0.287 & 0.510 & 0.983 \\
\hline 2011 & 0.286 & 0.564 & 0.736 \\
\hline 2012 & 0.284 & 0.277 & 0.822 \\
\hline 2013 & 0.290 & 0.316 & 0.945 \\
\hline 2014 & 0.298 & 0.372 & 0.930 \\
\hline 2015 & 0.309 & 0.440 & 0.814 \\
\hline 2016 & 0.324 & 0.517 & 0.712 \\
\hline
\end{tabular}



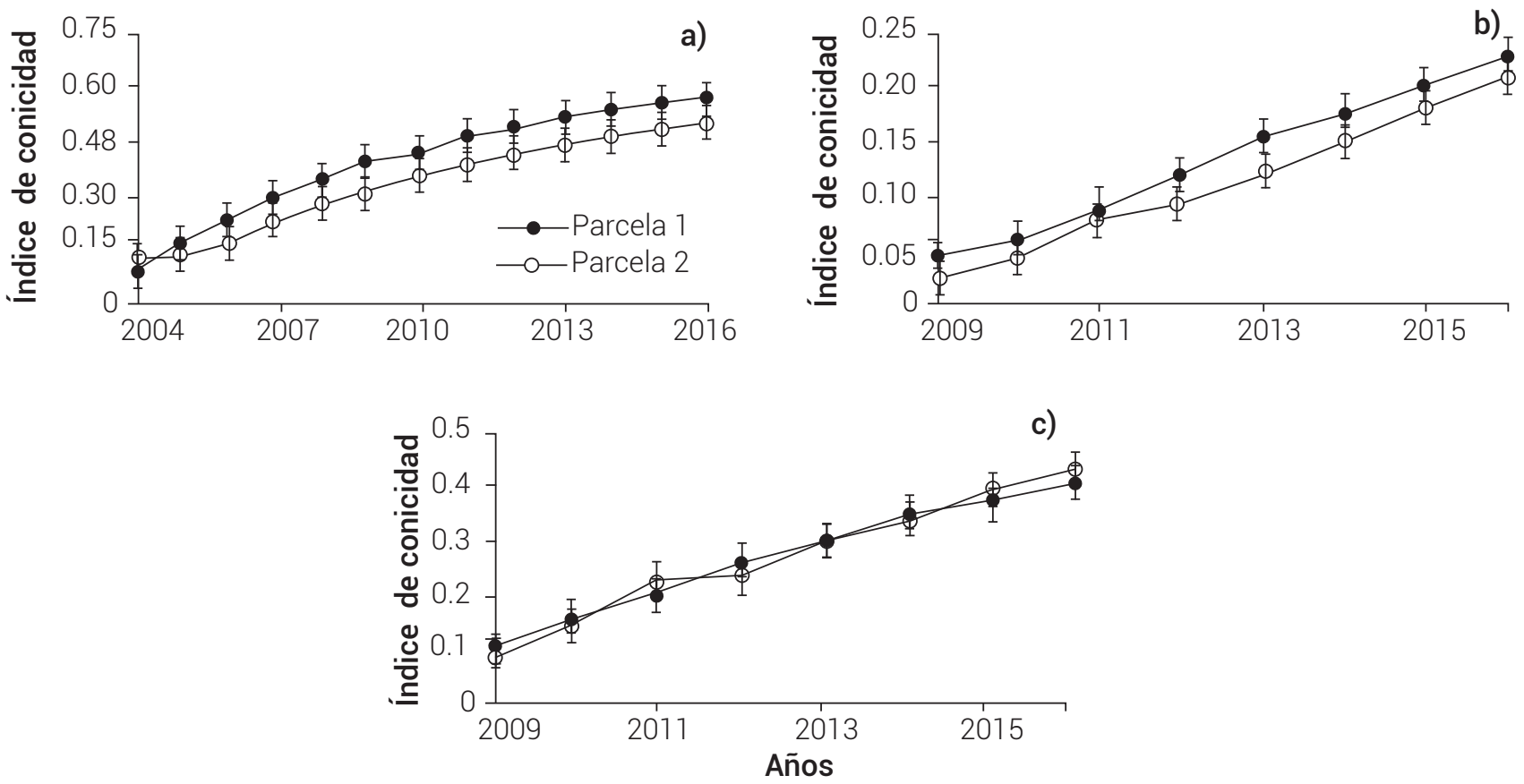

Figura 3. Índices de conicidad (IC) a diferentes alturas del fuste en parcelas aclareadas con diferente intensidad en una plantación de Pinus patula; a) IC $\mathrm{S}_{2} / \mathrm{S}_{1}\left(\mathrm{IC}_{21}\right)$, b) IC S $\mathrm{S}_{3} / \mathrm{S}_{1}\left(\mathrm{IC}_{31}\right)$, c) IC $\mathrm{S}_{3} / \mathrm{S}_{2}\left(\mathrm{IC}_{32}\right)$.

y modificar el patrón de incremento de los anillos de crecimiento. Existe una reducción drástica en el grueso del anillo y de los tipos de madera temprana y tardía en los años previos a la primera corta de aclareo, por la competencia excesiva de espacio entre los árboles en la plantación. La aplicación de cortas tardías de aclareo en Pinus patula con densidad elevada de árboles residuales redujo gradualmente el grueso de los anillos de crecimiento sin modificar la relación entre la madera temprana y tardía. El mayor grueso de los anillos en la parte intermedia y superior del fuste ocasionaron reducción gradual en la conicidad, lo cual indica la aplicación tardía de los aclareos, cuando ya había ocurrido la recesión de la copa debido a la elevada competencia por espacio.

\section{BIBLIOGRAFÍA}

Balducci L., A. Deslauriers, A. Giovannelli, S. Rossi and C. B. K. Rathgeber (2013) Effects of temperature and water deficit on cambial activity and woody ring features in Picea mariana saplings. Tree Physiology 33:1006-1017, https://doi.org/10.1093/treephys/ tpt073

Dvorak W. S., G. R. Hodge, J. E. Kietzka, F. Malan, L. F. Osorio and T. K. Stanger (2000) Pinus patula. In: Conservation and Testing of Tropical and Subtropical Forest Tree Species by the CAMCORE Cooperative. College of Natural Resources, North Carolina State University. Raleigh, North Carolina, USA. pp:148-173.

Daniels R. F., H. E. Burkhart and T. R. Clason (1986) A comparison of competition measures for predicting growth of loblolly pine trees. Canadian Journal of Forest Research 16:1230-1237, https://doi.org/10.1139/x86-218

Fernández M. P., J. Basauri, C. Madariaga, M. Menéndez-Miguélez, R. Olea and
A. Zubizarreta-Gerendiain (2017) Effects of thinning and pruning on stem and crown characteristics of radiata pine (Pinus radiata D. Don). iForest - Biogeosciences and Forestry 10:383390, https://doi.org/10.3832/ifor2037-009

Ferrere P., A. M. Lupi and T. Boca (2015) Crecimiento del Pinus radiata sometido a diferentes tratamientos de raleo y poda en el sudeste de la provincia de Buenos Aires, Argentina. Bosque 36:423-434 https://doi.org/10.4067/S0717-92002015000300009

Gartner B. L., E. M. North, G. R. Johnson and R. Singleton (2002) Effects of live crown on vertical patterns of wood density and growth in Douglas-fir. Canadian Journal of Forest Research 32:439-447, https://doi.org/10.1139/x01-218

Gruber A., S. Strobl, B. Veit and W. Oberhuber (2010) Impact of drought on the temporal dynamics of wood formation in Pinus sylvestris Tree Physiology 30:490-501, https://doi.org/10.1093/treephys/ tpq003

Hernández L. A., M. Martínez R., J. J. Vargas H., A. Velázquez M. y G. Ángeles P. (1996) Características de los anillos y densidad de la madera a diferentes alturas del fuste en rodales aclareados de Pinus patula Schl. et Cham. Ciencia Forestal en México 21:39-55.

Koga S., K. Oda, J. Tsutsumi and T. Fujimoto (1997) Effect of thinning on the wood structure in annual growth rings of Japanese Larch (Larix leptolepis). IAWA Journal 18:281-290, https://doi. org/10.1163/22941932-90001492

Larson P. R., D. E. Kretschmann, A. Clark III and J. G. Isebrands (2001) Formation and properties of juvenile wood in Southern pines: a synopsis. General Technical Report. Forest Service, United States Department of Agriculture. Madison, Wisconsin, USA. 42 p, https://doi.org/10.2737/FPL-GTR-129

Larson P. R. (1969) Wood formation and the concept of wood quality. Bulletin No. 74. School of Forestry, Yale University. New Haven, Connecticut, USA. $54 \mathrm{p}$

Niemistö P., H. Kilpeläinen and E. Poutiainen (2018) Effect of first thinning type and age on growth, stem quality and financial performance of a Scots pine stand in Finland. Silva Fennica 52:7816, https:// doi.org/10.14214/sf.7816

Pamerleau-Couture É., C. Krause, D. Pothier and A. Weiskittel (2015) Effect of three partial cutting practices on stand structure and growth of 
residual black spruce trees in north-eastern Quebec. Forestry 88:471-483, https://doi.org/10.1093/forestry/cpv017

PapeR.(1999) Effects of thinning regime on thewood properties and stem quality of Picea abies. Scandinavian Journal of Forest Research 14:38-50, https://doi.org/10.1080/02827589908540807

Pinkard E. A. and C. L. Beadle (2000) A physiological approach to pruning. The International Forestry Review 2:295-305.

Plomion C., G. Leprovost and A. Stokes (2001) Wood formation in trees. Plant Physiology 127:1513-1523, https://doi.org/10.1104/ pp.010816

Rodríguez-Ortiz G., A. Aldrete, V. A. González-Hernández, H. M. de los Santos-Posadas, A. Gómez-Guerrero y A. M. Fierros-González (2011a) ¿Afectan los aclareos la acumulación de biomasa aérea en una plantación de Pinus patula? Agrociencia 45:719-732.

Rodríguez-Ortiz G., V. A. González-Hernández, A. Aldrete, H. M. de los Santos-Posadas, A. Gómez-Guerrero y A. M. Fierros-González (2011b) Modelos para estimar el crecimiento y eficiencia de crecimiento en plantaciones de Pinus patula en respuesta al aclareo. Revista Fitotecnia Mexicana 34:205-212.

Rodríguez-Ortiz G., H. M. de los Santos-Posadas, V. A. González-Hernández, A. Aldrete, A. Gómez-Guerrero y A. M. Fierros-González (2012) Modelos de biomasa aérea y foliar en una plantación de pino de rápido crecimiento en Oaxaca. Madera y Bosques 18:25-41.

SAS Institute (2016) Base SAS® 9.4 Procedures Guide: Statistical Procedures. SAS Institute. Cary, North Carolina, USA. 576 p.

Sánchez-González A. (2008) Una visión actual de la diversidad y distribución de los pinos de México. Madera y Bosques 14:107120, https://doi.org/10.21829/myb.2008.1411222

Swidrak I., A. Gruber, W. Kofler y W. Oberhuber (2011) Effects of environmental conditions on onset of xylem growth in Pinus sylvestris under drought. Tree Physiology 31:483-493, https:// doi.org/10.1093/treephys/tpr034

Tong Q., F. Tanguay and S. Y. Zhang (2011) Impact of commercial thinning on annual radial growth and wood density in plantation-grown black spruce. Wood and Fiber Science 43:311-325.

Uranga-Valencia L. P., H. M. de los Santos-Posadas, J. R. Valdez-Lazalde, J. López-Upton y H. Navarro-Garza (2015) Volumen total y ahusamiento para Pinus patula Schiede ex Schltdl. et Cham. en tres condiciones de bosque. Agrociencia 49:787-801.

Vásquez G. I., M. A. López L., G. Ángeles P. and V. M. Cetina A. (2016) Growth and increase of a Pinus patula plantation with fertilization and thinning treatments. International Journal of Environmental and Agriculture Research 2:73-78.

Winck R. Á., H. E. Fassola y M. C. Área (2015) Efecto del raleo sobre las propiedades anatómicas de la madera de Pinus taeda. Maderas. Ciencia y Tecnología 17:391-406, https://doi.org/10.4067/ S0718-221X2015005000037

Zhang S. Y., G. Chauret, D. E. Swift and I. Duchesne (2006) Effects of precommercial thinning on tree growth and lumber quality in a jack pine stand in New Brunswick, Canada. Canadian Journal of Forest Research 36:945-952, https://doi.org/10.1139/x05-307 